\title{
Gender and COVID-19 global research agenda: priorities and recommendations
}

\section{Introduction}

Calls for greater recognition of and attention to the influence of sex and gender on health have been longstanding, and the need for this has only been amplified by the COVID-19 pandemic. Apart from the direct effects of biological sex and socially-constructed gender differences on COVID-19 morbidity and mortality - with higher rates of severe disease and deaths among men, pandemic responses have also amplified existing gender inequalities, with women bearing the heaviest burden of the indirect health and socio-economic consequences. The interactions between sex, gender and COVID-19 are complex and evolving, and further shaped and influenced by context and the intersecting influence of other social determinants and/or identities (such as race, ethnicity, LGBTQIA or migrant status, etc.), which have exacerbated the devastating health impacts for specific women, men and gender-diverse people. Recognising both the urgency of integrating sex and gender into COVID-19 research, and the roadblocks in the way of achieving this, the United Nations University International Institute for Global Health (UNU-IIGH) and the School of Public Health at the University of the Western Cape coconvened a collaborative gender and COVID-19 research agenda-setting exercise.

\section{Background}

From the outset of the COVID-19 pandemic, researchers, activists and policymakers highlighted the need to address and mitigate emerging and deepening gender inequalities in the crisis response. The differing impact of protective measures such as lockdowns on diverse groups, the unequal burden of unpaid care, and other inequities all underscored the dangers of a gender-blind pandemic response. In research and development, insufficient attention to biological sex and inclusion of women limited the impact and quality of research. The necessity of integrating sex and gender in order to produce quality science is still insufficiently recognised, and progress is limited by widespread sex and gender biases among decision-makers, combined with a weak evidence base, and incomplete data systems.

Under the coordination of WHO, the scientific community mobilized in an unprecedented way to identify research priorities and implement the COVID-19 Research Roadmap. Yet, sex-based differences and gender inequalities are insufficiently integrated in these research efforts, with implications for the quality of the science and the effectiveness of the tools being developed. As the world continues to respond to the evolving COVID19 crisis, sex and gender must be included in research investments informing immediate COVID-19 care and action, as well as long-term recovery. If not, scientific solutions will remain inadequate for a large part of the population, and we will not realise the transformation needed in how health systems serve their populations to accelerate health and well-being for all, in this and future pandemics.
Clinical Trials: Only $4 \%$ of $\underline{4,420}$ registered SARS-CoV-

2/COVID-19 studies explicitly reported a plan to include sex/gender for analysis. Only 8 of the 45 COVID-19 randomized controlled trials with results published by December 2020, reported sex-disaggregated results or subgroup analyses.

Health Services: Across 205 countries, only 17 report sex-disaggregated data on testing, 180 on confirmed cases, 74 on hospitalization, 19 on ICU admissions and 135 on deaths.

Health Policy: Across 334 task forces assessed, women made up on average $24 \%$ of the membership and only led $19 \%$ of them. 


\section{Methods}

The gender and COVID-19 research agenda-setting exercise was co-developed through real-time learning, and open calls with a broad range of stakeholders contributing to its design, scope and content, through webinars and a community discussion board (www.ghhbuzzboard.org). This crowd-sourced collaborative, anchored in the Global South, engaged with over 1000 participants from around the world through several steps, including five global meetings, four regional consultations, and nearly 500 participants in the online discussion board. Participants co-developed research questions and responded to surveys to rank each question according to urgency and impact on public health and on gender equality. The output is a shared, prioritised, policy-relevant and people-centred research agenda for civil society, programme implementers, policymakers, funders, and researchers that applies a gender lens to COVID-19 research investments and subsequently programming and policy actions.

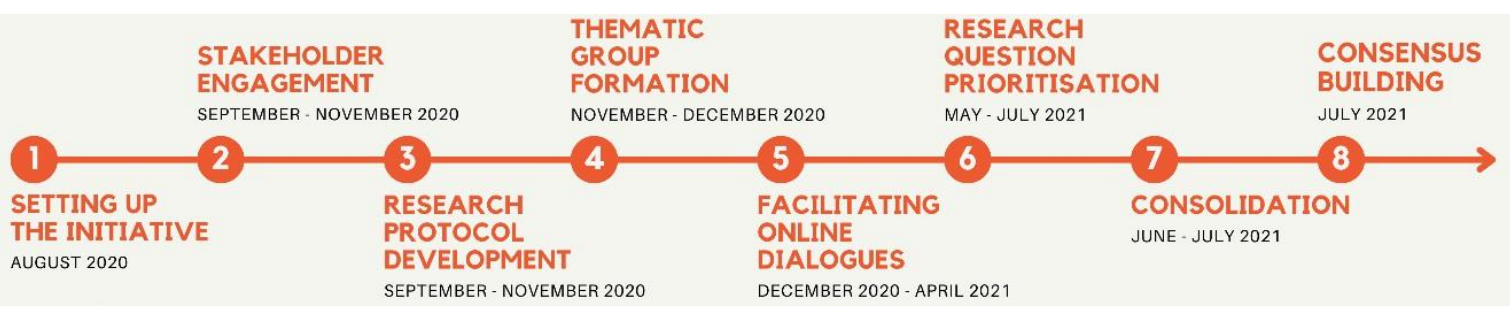

\section{Prioritised research questions}

The research agenda is comprised of five themes, spanning across the benches, beds and boardrooms of the health sector. For background information on each theme, read this brief.

\section{Health Status and Behaviour}

Sex and gender differences in COVID-19 risk, morbidity and mortality; the uptake of non-pharmaceutical interventions and vaccines; and impact on non-COVID health outcomes.

Acceptance and uptake of COVID-19 vaccines: Do the gender differences in the trust, acceptance and uptake of COVID-19 vaccines vary across social categories (such as race, disability, migrant status, age, sexuality and pre-existing conditions)

Acceptance and uptake of COVID-19 vaccines: Are there gender differences in the acceptance and uptake of COVID-19 vaccines?

COVID-19 infections, acute morbidity, and mortality: What are the infection, acute morbidity and mortality levels of COVID-19 among pregnant and post-partum women, and their foetus/infants across various contexts?

Post-COVID conditions: How do post-COVID conditions affect pregnant and postpartum women, and their foetus/infants across various contexts?

Mental Health and other NCDs: What was the impact of COVID-19 measures on the mental health outcomes of women, men, women, girls, boys, LGBTQI+ and gender-diverse persons?

\section{Research and development}

Integration of biological sex (female, male, intersex) and gender (women, men, gender-diverse individuals) into the research and development of COVID-19 prophylactic products (including vaccines), therapeutics, medical devices, diagnostics, and digital health interventions.

Participation \& engagement: How can pregnant and lactating females be ethically, and safely included in phase 3 and 4 studies for COVID-19 R\&D?

Regulation, funding \& commercialisation: In what way are sex and gender related variables integrated into national and global vaccine safety surveillance systems?

Therapeutics \& vaccines- specific population outcomes: Does safety, efficacy, optimal dosing regime, and protective duration of the different COVID-19 vaccines differ in pregnant and lactating women, and 
their foetuses and infants/toddlers?

Therapeutics \& vaccines outcomes: Does safety, efficacy and optimal dosing of different therapeutic interventions for COVID-19, and post-COVID conditions differ by sex, age, race?

Therapeutics \& vaccines-specific population outcomes: Does safety, efficacy and optimal dosing regimens of different therapeutic interventions for COVID-19, and post-COVID conditions differ in pregnant and lactating women, and their foetuses and infants/toddlers?

Participation \& engagement: What is the extent of the enrolment and participation of women in ongoing and completed COVID-19 clinical trials across various sites and countries?

Digital health: How can digital health intervention algorithms used in the pandemic be built to correct for gender and race bias?

\section{Health Service Delivery}

How gender influences the inputs for, quality of and utilisation of health service delivery for COVID-19 and non COVID-19 health conditions.

Access: How did health service delivery measures respond to the needs of pregnant women_who tested positive for COVID-19?

Access: To what extent, and how has, utilization of quality sexual, reproductive and maternal health and violence against women services changed because of COVID-19?

Access: How has the prioritization of COVID-19 services affected access to services for non-COVID-19 health conditions by gender and its intersection with other social categories?

Access: How does access and quality of services for COVID-19 differ by gender and its intersection with other social categories (such as race, disability, migrant status, age, sexuality, etc.) in various contexts?

Access: What strategies were used to improve gender and other inequities in access and quality of care for COVID-19 services (testing, facility-based care, quarantine care, etc.) and how effective were they?

Service delivery models: What are the different service reorganization models implemented to ensure continuity of maternal health, sexual, and reproductive and maternal health (SRHR) and violence against women and girls (VAWG) services during the pandemic, and how effective are they?

\section{Social and structural determinants}

The impact of the COVID-19 pandemic on specific social and structural determinants of health with strong gendered manifestations, including gender-based violence; skewed gendered income, occupation, labour, and unpaid care; social norms; education; and the environmental determinants of health.

Gender-based violence: How has the prevalence, incidence, severity, and frequency of different types of GBV (including online violence, and child marriage) changed during the different phases of the COVID-19 pandemic? Gender-based violence: Which women and girls facing intersecting forms of discrimination (including age, poverty, disability, and sexuality, etc.) are the most vulnerable to, and affected by, different types of GBV during the pandemic?

Gender-based violence: What are the determinants and pathways of increased GBV in the context of COVID-19? Gender-based violence: What policies, programmes and interventions have been successful and most costeffective in preventing GBV during the pandemic, and over the long-term?

\section{Gender and health governance for COVID-19}

Understanding relationships across actors influencing power and decision making in health systems

Data \& Research Governance: How to best support national statistical systems to produce and use sex and gender data during COVID-19 and future pandemics?

Gender mainstreaming: What do responsive and resilient health systems that address gender bias and advance gender equality look like?

Gender mainstreaming: To what extent, and how, is gender considered in the current decision making and learning processes for COVID-19? 


\section{Most important priorities}

While theme four on social determinants was the theme with the most participation, questions related to research and development; health status and behavior; health service access and organization were prioritized as the most urgent for public health \& gender equality. The question ranked as most important was: 'How can pregnant and lactating females be ethically, and safely included in phase 3 and 4 studies for COVID-19 research and development?'

Questions about pregnant and lactating women were prioritized across themes 1, 2 and 3, indicating significant research gaps for this group. Biological differences remain an unaddressed priority for vaccine and therapeutics R\&D. Sex disaggregated analysis in clinical trials, safety surveillance systems and basic health management information systems must be prioritized further. In addition, research that examines and addresses the power dynamics that frame the lived realities of those most marginalized were also highly valued, in particular evidence to understand and respond to gender-based violence in the context of COVID-19, and policy analysis of gender mainstreaming in COVID-19 responses for accountability.

\section{Recommendations}

$>$ Integrate sex and gender into existing research investments \& platforms

$>$ Increase investment in high-impact and high-quality research that addresses:

$0 \quad$ Sex and gender in vaccine and therapeutics $R \& D$

- Real-time research on vaccine hesitancy and uptake

- Indirect and long-term impacts on health and wellbeing, including GBV, mental health

- Implementation research to design, evaluate and learn from gender-responsive policies, responses and adaptations in health service delivery that promote gender equality or mitigate gender inequalities

- Research that supports multi-sectoral action to address to gendered social determinants and consequences of COVID-19 on those most marginalized

0 Research that reveals and transforms the gender power dynamics in health system decision making for COVID-19

\section{Resources}

- https://www.ghhbuzzboard.org/ - the online discussion board for the gender and COVID-19 research agenda-setting exercise, and repository for presentations, blogs and articles.

- https://www.genderhealthhub.org/articles/what-why-and-how-gender-covid-19-research-agendasetting/ - explainer brief on the background and process of the research agenda-setting exercise.

- https://www.genderhealthhub.org/articles/guidance-note-and-checklist-for-tackling-gender-relatedbarriers-to-equitable-covid-19-vaccine-deployment/ - a guidance note and checklist of key actions for countries to prioritise in the planning, delivery, and monitoring of vaccine deployment.

- https://www.genderhealthhub.org/?loc=all\&cate=13\&s - all work from UNU-IIGH Gender and Health Hub on COVID-19.

- https://www.who.int/teams/blueprint/covid-19 - WHO R\&D Blueprint.

- https://www.who.int/emergencies/diseases/novel-coronavirus-2019- WHO research and information on COVID-19.

- $\quad$ https://www.genderandcovid-19.org/gender-working-group-page/ - gender and COVID-19 working group, sharing information, resources and insights.

- https://data.undp.org/gendertracker/ - the COVID-19 Global Gender Response Tracker monitors responses taken by governments worldwide to tackle the pandemic, and highlights those that have integrated a gender lens.

- https://globalhealth5050.org/the-sex-gender-and-covid-19-project/ - tracking differences in COVID-19 infection, illness and death among women and men and analysing inclusion of sex and gender in national COVID-19 health policies. 